\title{
Anatomical Differences between Children and Adults
}

\author{
${ }^{1}$ Rengin Kosif, ${ }^{2}$ Rabia Kecialan* \\ ${ }^{1}$ Bolu Abant Izzet Baysal University, Medical Faculty, Department of Anatomy, Bolu, Turkey \\ ${ }^{2}$ Bolu Abant Izzet Baysal University, Health Sciences Faculty, Department of Pediatric Nursing, Bolu, \\ Turkey
}

\begin{abstract}
In this review, anatomical differences between child and adult were mentioned. These differences are especially apparent in infancy and preschool term. When the child reaches the school age term, the differences begin to decrease gradually. When the child reaches the age of 18 , the child has the same characteristics as the adult. The main differences include skin, subcutaneous tissue, total amount of water in the body, muscles preferred in pharmaceutical applications, external ear structure, Eustachian tube, anatomy of the eye, bone skeletal structure, spinal cord and brain, respiratory tract, digestive organs, cardiovascular system and urinary system. The differences especially between child and adult brain structure are striking. The brain tissue in the child is more sensitive, calvarium is thinner, subarachnoid space is narrower. Morover; the differences in gynecological examination and lumber puncture practices were also reviewed. In adults, gluteal muscles are used in intramuscular applications, while in infants, rectus femoris and vastus lateralis muscles are commonly used. These anatomical differences are important for the diagnosis and treatment of the doctors. Nurses should take these differences into account in pediatric drug applications in the clinic and in the care of children. Clinicians should know that children are not small adults.
\end{abstract}

Keywords: Pediatric Anatomy, Adult Anatomy, Differences, Tissues and Organs.

\section{Introduction}

There are several major anatomical differences between children and adults that can affect your assessment and treatment. These differences are more pronounced in younger children (infant through preschool age); they begin to disappear as the children age into school age and adolescence. By the time they are 18, most of the changes are complete [1].

According to recent clinical findings, infant subcutaneus tissue is $30 \%$, and infant epidermis $20 \%$ thinner than in adults. Consequently, baby skin is more permeable and more prone to dryness than adult skin. Furthermore, a baby's body surface to volume/weight ratio is higher than that of adults, which increases baby skin's vulnerability to applied substances and to sun exposure. In children the sebaceous glands are large, in adults they are small. Fat tissue is higher in infants and less in adults. Fat mass as a proportion of body weight is also higher in infants. Percent body fat in humans peaks between 3-6 months of age. Nails are soft and thin in childhood, hard and thick in adults [2].

Total amount of water is $75-80 \%$ of body weight in childhood, $60 \%$ in adults. Neonates and infants lose heat more rapidly than adults because the surface area on their heads is larger by comparison to their body mass. Also, young children have an undeveloped hypothalamus, which means that their ability to regulate temperature is impaired [3].

The administration of injections into the rectus femoris or the vastus lateralis is a common practice among paediatric and family care nurses (Figure 1). Due to the larger muscle size in the anterolat-eral thigh, the vastus lateralis muscle is the recommended site for intramuscular (IM) injections in infants $<12$ monthsof age, but the deltoid muscle is recommended for IM injections in children $\geq 12$ months. While the most commonly referenced IM injection site for infants of less than 12 months is vastus lateralis (VL) as it has advantages such as ease of access and adequate muscle mass. The muscle in the ventrogluteal site is 
adequately developed, even in infants between the ages of 1-12 months and that in particular, in children 1236 months old, the ventrogluteal site is even thicker than the anterolateral [4].

\section{Figure 1: Vastus Lateralis Injection Site on the Anterolateral Thigh in Children}

(https://immunisationhandbook.health.gov.au/resources/handbook-figures/figure-vastus-lateralis-injectionsite-on-the-anterolateral-thigh)

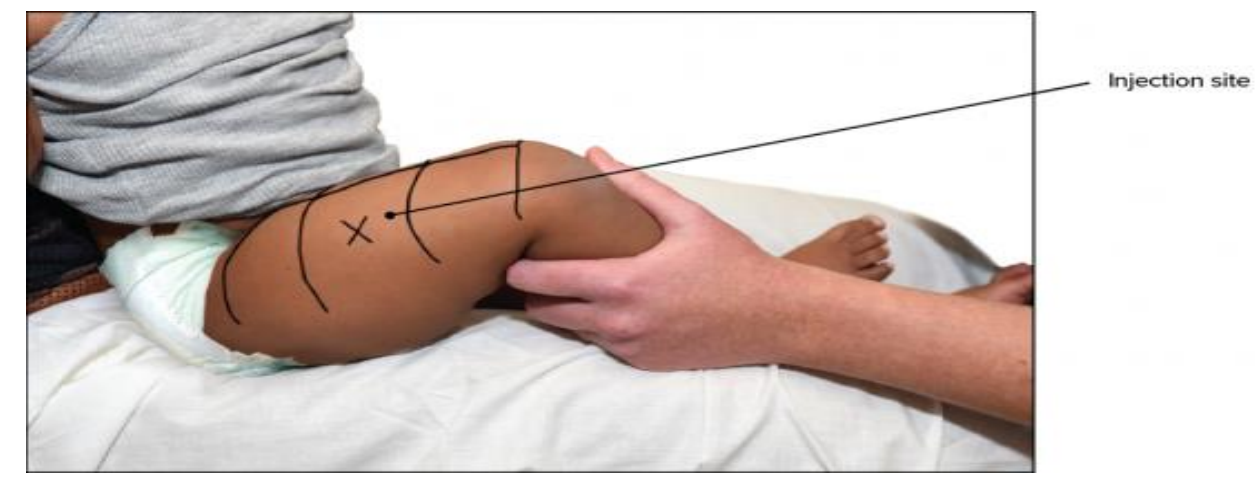

Injections sites in the hip area, such as the gluteal muscles, are preferred for the administration of larger volumes of medications, while small volumes can be given to the arm, in the deltoid muscle in adult (Figure 2) [5].

\section{Figure 2: Most Commonly Used IM Injection Site in Adults}

(http://nursingfile.com/nursing-procedures/demo-video/how-to-administer-dorsogluteal-injection.html)

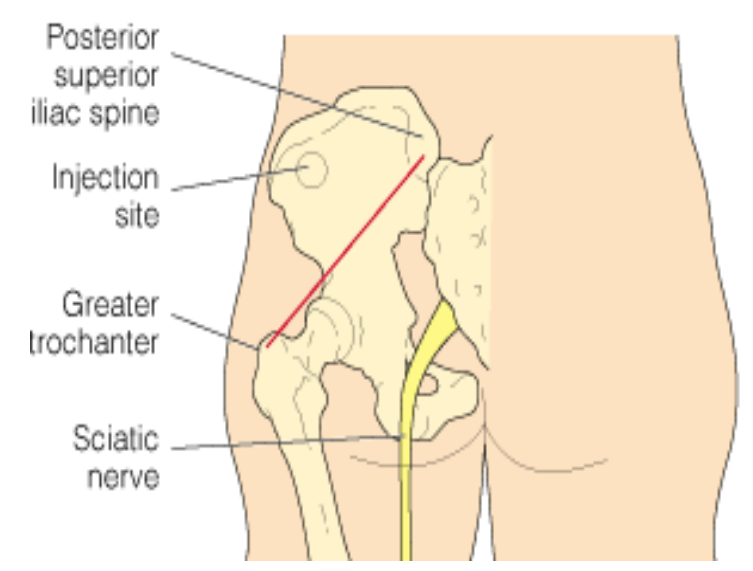

The external auditory canal (EAC) curves inward and is approximately $24 \mathrm{~mm}$ long. Cartilage surrounds the outer portion and the skin is hairy and contains glands that produce cerumen. The inner portion is surrounded by bone and lined with thin, hairless skin. In infants, the EAC is directed upward and backward from the outside. The EAC curves downward and forward in children $>3$ years of age. Grasp the auricle and firmly but gently pull it upward, backward, and slightly away from the head (straightens the EAC, should be painless). The EAC curves upward in infants so pull down and back to the 6 to 9 o'clock range. The EAC curves downward and forward in children $>3$ years of age so pull up and back toward a 10 o'clock position. The Eustachian tube is short and straight in children, long and curved in adults [6].

Their retinal images are also smaller compared to adults due to shorter distances from the retina to the cornea of the infants' eye. A newborn's pupil grows from approximately $2.2 \mathrm{~mm}$ to an adult length of 3.3 $\mathrm{mm}$. It is important to note that the distance from the cornea at the front of the infant's eye to the retina which is at the back of eye is $16-17 \mathrm{~mm}$ at birth, 20 to $21 \mathrm{~mm}$ at one year, and $23-25 \mathrm{~mm}$ in adolescence and adulthood. This results in smaller retinal images for infants. Color sensitivity improves steadily over the first year of life for humans due to strengthening of the cones of the eyes. The threshold for light sensitivity is much higher in infants compared to adults. From birth, the pupils of an infant remain constricted to limit the amount of entering light. In regards to pupil dimensions, newborn's pupil grow from approximately 2.2 $\mathrm{mm}$ to an adult length of $3.3 \mathrm{~mm}$ [7]. 
Figure 3: Child And Adult Skeletal System (https://health.clevelandclinic.org/growth-plate-injuries-know/)

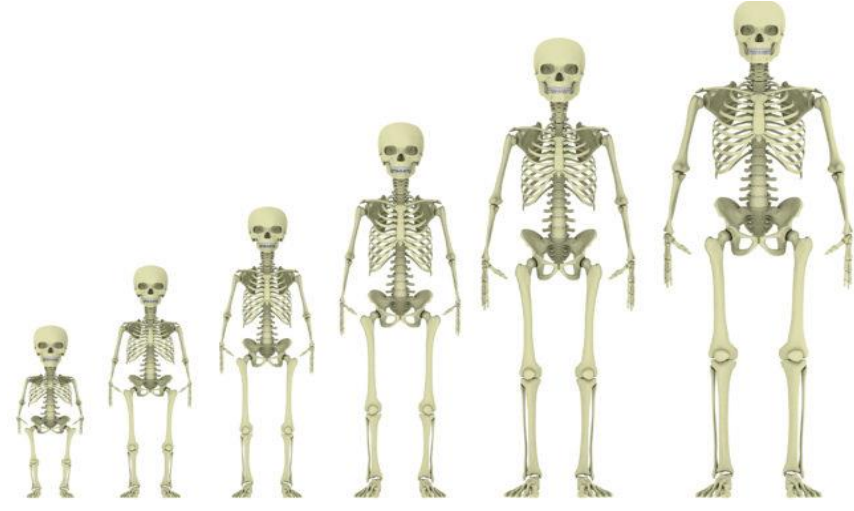

Relative to the size of a child's body, the head is large and heavy, balanced on a neck poorly supported by weak muscles and ligaments, and so both head and cervical spine are easily injured (Figure 3). Biomechanical maturation of the spine is a progressive process that only starts to resemble the adult spine after age 8-9 years old. Epiphyses fuse at different times and are easily mistaken for fractures. The pattern of injuries is determined by these progressive changes. Most spine injuries in children occur in the cervical region; in younger patients, these are more often subluxations or dislocations, more often in the upper cervical spine, and more often associated with neurological injury. The calvarium is thin in young children; this, with the sutures and fontanels, allows for easy deformation, with or without fracturing, under external pressure [8]. More fragile brain tissue, Thinner subarachnoid space, Brain demands greater amounts of oxygen and glucose [1].

Young children are at particularly high risk of airway obstruction. Their tongues are relatively large for their oral cavity, as are the soft palate and soft tissues of the mouth and the epiglottis, which is relatively longer and stiff. They also have a larynx that is higher and more anterior, a cricoid ring that represents the narrowest point of the airway, and a shorter trachea that bifurcates higher. The trachea has a small diameter and is compressible. Respiration rate is faster than adult. The respiratory mucosa is very gentle, it does not produce mucus [9].

Liver and spleen are lowering level and less protected by ribcage. In children, the liver is large, has more space in the abdominal cavity, has less space in the adult. Less well protected by undeveloped abdominal muscles. Proportionally greater body surface area than adults. Proportionally less fat; thinner skin layers. (Arthur) Tonsillae are larger and produce antibodies in chidhood, smaller in adults. Tonsils and adenoids grow disproportionately fast in children, making any inflammatory response more likely to compromise the movement of air. Tonsillitis can be a life threatening infection for a child as opposed to an annoyance to an adult [10].

In children, the tongue is large and the mouth and nasal cavity are small. In adults, the tongue is small and the mouth and nose cavity are large. Cardia sphincter is loose in children, tight in adults. In children, stomach emptying is fast and slow in adults [11].

Children generally have very good cardiovascular health. This enables them to compensate well for circulatory problems such as hypervolemia and dehydration. There will rarely be a change in a child's blood pressure until it is about to have a cardiac arrest. The heart rate of a child makes a better diagnostic tool.

- A child has much small upper and lower airways which results in a great chance of respiratory difficulties and failure.

- Infants are abdominal breathers who rely primarily on the muscles of the diaphragm. This means abdominal distension can lead to respiratory problems.

- The immature muscles associated with respiration, such as the diaphragm, intercostal muscles and sternocleidomastoid are more likely to fatigue.

- The respiratory centre is relatively immature. This means that neonates and young infants have irregular respirations and are at a greater risk of apnoea. Many neonates will literally feed and forget to breathe. 
- An infant is an obligatory nose breather for the first 6 months, which means that a blocked nose can lead to respiratory failure.

- Infants have very short and softer tracheas than adults. This means that overextension during airway manoeuvres may result in airway collapse

- Infants have proportionately large heads, short necks and large tongues, which again, makes airway obstruction more likely.

- Both infants and even toddlers have a long and floppy epiglottis, which means more chance of airway obstruction [3].

In adults, breathing requires the coordinated use of three muscle groups, the diaphragm, intercostal muscles (the muscles between each rib) and the abdominal muscles. Babies and young children will use their abdominal muscles much more to pull the diaphragm down for breathing. The intercostal muscles are not fully developed at the time of birth. Breathing rates in resting adults can range from 10-15 times per minute. Neonates: 30-60/minute, Infants: 30-40/minute, Toddlers: 20-40/minute [12].

In children, the bladder is in the abdominal cavity when empty and full. The bladder is an abdominal organ and is less protected by the pelvis in young children. 6. at age the bladder begins to enter the pelvis major. In puberty the bladder takes its place in adults. It is inside the pelvis minor when the bladder is empty. It can rise up to the umbilicus while the bladder is totally full. Urinary bladder capacity is low and affected by stress in childhood. Urinary bladder capacity in adults is $500 \mathrm{cc}$ maximum and is not affected by stress [13].

A full gynecologic examination of the child mainly includes inspection of external genitalia and in some cases rectal examination. Performing vaginoscopy and obtaining samples are limited to certain cases. Most important is a deep anatomical and physiological knowledge of the genital system in the child, including the learning of nonaggressive examination technical skills. It involves a nonjudgmental approach to the child and adolescent gynecological patient, which will allow the development of a trusting relationship between a confident patient and a supportive physician [14].

Pediatric lumbar punctures (LP) can have much higher success rates when proper positioning and technique are emphasized. The position should be the lateral decubitus position with hips flexed, but the neck does not need to be flexed. The spinal cord ends at L3 in newborns so the lumbar puncture should be performed at the top of the iliac crest, L3/4 or L4/5 intervertebral space. Usually, the L2-3, and L3-4 levels are preferred for LP in adults [12].

Medical doctors and nurses should take into account the above-mentioned anatomical differences in medical processes. Clinicians should know that 'children are not little adults' before starting the inspection.

\section{The authors declare that there is no conflict of interest regarding the publication of this article.}

\section{References}

[1.] Hsieh A. (2014) Emergency Medical Technician With Online Practice. ISBN: 978-1-118-76817-4. Pg: 288, e-book.

[2.] Eichenfiel L, Frieden I, Mathes E, Zaeglein. (2014) A Neonatal and Infant Dermatology ISBN: 9781455726394. 3. Edition. Pg: 56, e-book.

[3.] Emergency Medical Paramedic 2010-2018. Anatomical and Physiological Differences in Children. http://www.emergencymedicalparamedic.com/anatomical-and-physiological-differences-inchildren/

[4.] Yapucu U, Ceylan GB, Ceylan B, Bayındır P. (2015) Is The Ventrogluteal Site Suitable For İntramuscular İnjections İn Children Under The Age Of Three? Journal of Advanced Nursing. 72 (1): 127-133.

[5.] JoVE Science Education Database. (2020) Nursing Skills. Preparing and Administering Intramuscular Injections. JoVE, Cambridge, MA, https://www.jove.com/scienceeducation/10261/preparing-and-administering-intramuscular-injections

[6.] Bickley LS. (2013) Bates' Guide to Physical Examination and History Taking. 11th ed. Philadelphia, PA: Lippincott Williams \& Wilkins. ISBN-10: 1609137620. Pg: 232-6, 834-5. 
[7.] Thomasson MA, Teller DY. (2000) Infant color vision: Sharp Chromatic Edges Are Not Required For Chromatic Discrimination İn 4-Month-Olds. Vision Research. 40 (9): 1051-1057.

[8.] Figaji AA. (2017) Anatomical And Physiological Differences Between Children And Adults Relevant To Traumatic Brain Injury And The Implications For Clinical Assessment And Care. Front Neurol. 8 (685): 1-15.

[9.] Bliss D, Silen M. (2002) Pediatric Thoracic Trauma. Crit Care Med. 30: 409-15.

[10.] Zanin C, Perrin P, Bene MC, Perruchet AM, Faure GC. (1994) Antibody-Producing Cells İn Peripheral Blood and Tonsils after Oral Treatment Of Children With Bacterial Ribosomes. International Journal of Immunopharmacology. 16 (7): 497-505.

[11.] Henretig FM, King C. (1997) Textbook Of Pediatric Emergency Procedures. By Williams \& Wilkins. Pg: 162.

[12.] Esheric J. (2016) Hospital Procedures Consultant, Differences Between Lumbar Punctures İn Children Vs Lumbar $\quad$ Punctures https://www.hospitalprocedures.org/blog/view/1272/differences-between-lumbar-punctures-inchildren-vs-lumbar-punctures-in-adults

[13.] Marx J, Walls R, Hockberger R. (2013) Rosen's Emergency Medicine - Concepts and Clinical Practice. 8. Edition. Pg: 2094, e-book.

[14.] Cheikhelard A, Chaktourac Z, Thibauda E. (2012) Gynecologic Clinical Examination of the Child and Adolescent. Endocr Dev. 22: 1-10. 